\title{
ÉTUDE BIOLOGIQUE ET SYSTÉMATIQUE \\ DES SARCOPHAGINES MYIASIGĖNES \\ DU GENRE WOHLFAHRTIA
}

\author{
Par E. SÉGuY
}

Le présent travail donne un résumé des actions des espèces du genre Wohlfahrtia dont les larves présentent de remarquables tendances parasitaires. Il fera connaître une forme nouvelle dont les caractères distinctifs, très nets, ne paraissent pas fixés et laissent l'impression d'une espèce en évolution actuelle. De tels exemples sont utiles à connaître. L'étude qui suit aura pour conclusion un synopsis des espècesconnues qui permettra l'identification des Wohlfahrtia de l'Ancien Monde.

\section{Biologie.}

1. Période d'apparition des adultes. - En Europe centrale et méridionale, ainsi que dans le nord de l'Afrique, les Wohlfahrtia ne se montrent pas avant le commencement de juin. L'éclosion des adultes se poursuit jusqu'au début de septembre. Pendant toute cette période, on les rencontre surtout dans les endroits découverts, champs, prairies, pâturages, où ils volent rapidement. Ils s'approchent des lieux habités sans chercher à entrer dans les maisons, mais ils suivent l'homme et les animaux. Brauer a dit que ces mouches recherchaient les charognes, les viandes mortes et les matières en décomposition. Il est vrai qu'elles peuvent être attirées par l'odeur de ces substances, mais les Wohlfahrtia sont également des parasites actifs qui poursuivent les animaux vivants qui portent des plaies plus ou moins odorantes. L'homme est surtout attaqué pendant le sommeil : les individus qui dorment en plein air ou les nomades des déserts et des steppes, ou certains travailleurs agricoles, sont exposés aux attaques de ces Diptères.

En Russie, les animaux sont particulièrement poursuivis par le W. magnifica.

Selon Portchinsky, les Wohlfahrtia seraient dangereux aussi comme vecteurs de germes pathogènes en période d'épizootie. Mais ansales de Parasitologie, T. XVIII, Nor 4-5-6. - 1941, p. 220-232. 
d'après Jausion et Dekester, la transmission des kystes d'amibes par les mouches serait très limitée.

L.es Wohlfahrtia n'existent pas dans le nord de la France, en Angleterre, en Hollande, au Danemark, dans toute la péninsule scandinave, en Finlande, et dans les régions baltiques orientales et septentrionales. Dans ces régions, les animaux sont attaqués par le Lucilia sericata.

2. Nourriture et vie de l'adulte. - L'appareil buccal rappelle celui de la mouche domestique, mais il est beaucoup plus robuste. Les Wohlfahrtia se nourrissent des substances les plus diverses, liquides sucrés, humeurs suintant des plaies des végétaux ou des animaux, matières en décomposition, pollen des fleurs. On peut les trouver, avec d'autres Sarcophagides, sur les ombelles de certaines plantes.

3. Accouplement. - L'accouplement a lieu très peu de temps après l'éclosion et peut se produire pendant toute la durée de l'apparition des adultes. Il ne diffère pas essentiellement de celui que l'on peut observer chez les Sarcophaga ou les Calliphorides supérieurs.

4. Ponte. - La ponte des Wohlfahrtia a lieu en plein jour, entre dix et seize heures, de préférence pendant les journées chaudes et orageuses. Les femelles donnent des larves toutes du même âge ; elles les déposent par groupes de dix ou plus. I 20 ou r 70 larves peuvent ètre pondues par une même femelle, en une seule fois ou par petits groupes, sur les substances ou sur les organes des animaux qui doivent assurer l'évolution.

5. La larve. - C'est un petit ver opalin, couvert d'une peau dure armée de onze ceintures transverses épineuses. L'armature buccale, très robuste, présente une dent médiane saillante. Les organes antenniformes sont bien développés et les stigmates postérieurs sont placés sur de petites protubérances parfois rétractiles ou enfoncées dans une caverne stigmatique. Aux âges suivants, la peau s'épaissit, les épines, très nombreuses, robustes et triangulaires, réparties en plages étendues, couvrent presque toute la surface du corps. La dent médiane de l'appareil buccal disparaît; les stigmates antérieurs, profondément rétractiles, et les stigmates postérieurs, placés au fond d'une caverne, sont protégés par un bourrelet épais formé par un repli circulaire du tégument; cette disposition permet à l'animal de protéger les orifice stigmatiques et de soustraire l'appareil respiratoire à l'action des liquides nocifs. 
Selon Portchinsky, la larve peut survivre deux heures en immersion dans une solution à 2 p. I 000 de sublimé corrosif ou dans l'alcool à $95 \mathrm{p}$. roo; environ r heure 30 dans une solution à $5 \mathrm{p}$. roo d'acide borique; elle résiste à peu près trente minutes dans une solution d'acide phénique à $2 \mathrm{p}$. Ioo et deux minutes dans une solution à 5 p. Iоo. Elle peut rester dix minutes immergée dans l'acide chlorhydrique pur, mais elle meurt en une minute dans l'éther sulfurique ou dans l'eau chloroformée à $3 o$ p. ıoo.

Une larve qui était restée près d'une heure dans l'alcool à $95^{\circ}$, retirée et placée ensuite sur de la terre, s'est transformée en pupe qui a donné l'adulte. J'ai pu vérifier l'exactitude de ces données à quelques minutes près.

6. Evolution larvaire. - Le développement larvaire est soumis aux divers facteurs d'humidité, de chaleur et de nourriture. La nourriture et l'humidité sont indispensables, la larve peut subir un abaissement de température assez brusque sans que celui-ci ait des conséquences fatales. La grande résistance de ces asticots explique que les adultes capturés errants dans la nature soient d'une taille remarquablement constante. On n'observe pas chez les Wohlfahrtia cette souplesse morphologique qui paraît avoir été portée à son maximum chez certains xérophiles du genre Anthrax (Bombyliides) ou Sarcophaga (Calliphorides). Chez les Wohlfahrtia, diptères agressifs, à ponte aocélérée, les malingres disparaissent, soit à l'état larvaire $\left(2^{\mathrm{e}}\right.$ âge), soit à l'état pupal.

Le développement de quelques espèces a été étudié par M. Johannsen et par M. Walker. Les larves des Wohlfahrtia sont des animaux créophiles et saprophages qui peuvent provoquer des myiases graves avec la plus grande facilité. Dans les régions habitées par les mouches, les animaux portant de petites plaies sont attaqués par les Wohlfahrtia. Ces plaies, infestées par leurs larves, s'élargissent et s'aggravent. D'après Portchinsky, la plupart des cas de myiase humaine attribués à diverses espèces de Calliphora et de Sarcophaga seraient dus au Wohlfahrtia magnifica.

Les animaux les plus différents sont recherchés par les Wohlfahrtia. On a observé leurs larves sur le renard (Kingscote), le lapin (Shannon), le rat (Morrison), le cheval, le mouton, le bœuf et le chameau (Lütje, Delanoë), le porc, le chien, les volailles, surtout les oies (Portchinsky, Roubaud). Une seule espèce attaquerait des invertébrés. Les criquets des genres Nomadacris, Schistocerca et Locustana sont parasités, selon M. Faure, par le Wohlfahrtia pachytyli.

L'homme est attaqué de différentes façons, mais c'est surtout la 
région céphalique qui semble le lieu d'élection pour la plupart des Wohlfahrtia. Le nez, la bouche et les oreilles renferment souvent des larves. Les larves trouvées dans les oreilles ont été signalées par Blanchard, Condorelli, Fairmaire, Francaviglia, Gough, Lewis, Macias, Manliewicz, Najera, Popov, Selezneva et Taschenberg. Gerstäcker, Hagen, puis Laboulbène les ont trouvées dans les fosses nasales; elles ont été vues dans la cavité buccale par Brandt, Volkowicz et Cros. Les larves observées dans les yeux par Gough, Gerston et Goldschmidt sont peut-ètre des émigrantes provenant du nez. Elles auraient été trouvées dans l'intestin par Perroncito. Mais il semble que les cas de myiases intestinales par larves émigrantes ou ingérées soient très accidentels. Les myiases généralisées, attribuées aux Wohlfahrtia par Dubois, Onorato, Patton, Portchinsky et Walker, ont probablement pour cause une mouche d'un autre genre (Calliphora).

On a vu que les larves des Wohlfahrtia peuvent se trouver non seulement sur les animaux, dans les ouvertures ou les cavités naturelles, dans les plaies déjà existantes, mais encore dans les creux ou les plis tégumentaires où s'accumulent des matières sébacées qui fermentent rapidement. Les larves provoquent l'ulcération de la peau, pénètrent en dessous et leur action continue amène des désordres variés. Elles peuvent se trouver également sur les muqueuses, principalement sur les organes génitaux des vaches et des chamelles où elles séjournent longtemps dans des plaies qui ne guérissent pas. Chez les chiens, les larves des Wohlfahrtia s'observent particulièrement dans les oreilles. Les petits mammifères (rongeurs ou carnassiers) attaqués par ces mouches présentent rapidement des accidents mortels. Les larves des Wohlfahrtia achèvent leur développement dans le cadavre. M. C. Dumont a observé le fait sur des gerbilles de Tunisie.

La facilité avec laquelle les larves des Wohlfahrtia se développent dans les plaies a suggéré de les utiliser, comme les larves de Lucilia, pour le traitement de certains cas d'ostéomyélite. Une étude sur ce sujet a été publié par M. Grantham-Hill.

7. Nymphose. - La nymphose est semblable à celle des Myodaires supérieurs. Arrivée à son complet développement, la larve quitte le milieu où s'est effectuée son évolution, tombe sur la terre et se métamorphose dans le sol à peu de distance de la surface. La cuticule nymphale paraît ridée par la présence des épines cuticulaires érigées par le rétrécissement du tégument. La durée de la transformation, soumise aux influences extérieures, varie entre 7 et I $2_{2}$ jours. Au terme de cette période, la mouche sort de la peau nymphale. 
M. Ferrière a fait connaître un parasite de la pupe du Wohlfahrtia nuba : c'est un Chalcidien, le Dirhinus wohlfahrtiæ.

\section{Etude systématique du Wohlfahrtia nuba.}

Dans son étude sur les Wohlfahrtia, le $\mathrm{D}^{\mathrm{r}} \mathrm{H}$. Salem assigne les caractères suivants au W. nuba:

Fémurs II sans peigne apical postéro-interne. Dorso-centrales $\mathrm{I}+2$; supraalaires 3. Abdomen : tache centrale du tergite IV avec un faible prolongement antérieur. Forceps externe à branches en massue courte étranglée à la base.

Le $W$. nuba ainsi défini représente la forme typique de l'espèce telle qu'elle est décrite par H. Salem. Mais si l'on observe une longue série de ces insectes, on arrive par passage aux définitions suivantes :

I. - Fémurs II avec un peigne formé d'épines robustes placé dans la région apicale et postéro-interne. Dorso-centrales $2+2$; supra-alaires 2. Abdomen : tache centrale du tergite IV sans prolongement antérieur. Forceps externe à branches en massue courte, étranglée à la base.

II. - Fémurs II sans peigne épineux. Forceps externe à branches formées par une plaque rectangulaire non étranglée à la base.

Il faut remarquer que le $\mathrm{D}^{\mathrm{r}}$ Salem, dans sa description, confond les différentes formes. Les caractères chromatiques, l'apparence générale de l'appareil copulateur autorisent les confusions. Dans le tableau dichotomique des espèces, $H$. Salem place le $W$. nuba parmi les formes armées d'un peigne fémoral (p. 15 et 16$)$; dans la description de l'espèce ( $p .30)$, le fémur est inerme.

Pour élucider cette question et préciser les caractères spécifiques du $W$. nuba, il fallait procéder à plusieurs dissections. J'ai toujours constaté que les espèces à fémur inerme, souvent de petite taille, à pilosité réduite, portent un appareil copulateur dont les forceps externes sont quadrangulaires; que les formes dont le fémur II est armé d'un peigne sont de plus grande taille, et que les forceps externes de l'appareil copulateur sont en forme de massue courte, étranglée à la base, comme il est représenté sur la figure 5 du travail de H. Salem. Il y a encore des formes de passage dont les fémurs ont une tendance à s'armer et dont l'appareil copulateur porte des forceps externes à branches subquadrangulaires. Les différentes formes du Wohlfahrtia nuba s'agrègent au groupe smarti-indigens. 
Voici les caractères des deux formes extrêmes confondues sous le nom de Wohlfahrtia nuba. On retiendra comme type du W. nuba la forme dont l'appareil copulateur mâle a été figuré par H. Salem, p. 29-3 I, fig. $5 a$ et $b$. Dans les collections du Muséum de Paris, cette forme présente les caractères suivants.

Wohlfahrtia nuba (Wied.) Salem, Egypt. Univ. Publ. 13, p. 29, fig. $5 a$ et $b$. $\sigma^{x}$. - Tête. - Espace interoculaire subégal au tiers de la largeur de la tête vue de face $(8: 2,5)$. Bande médiane frontale blanche, subégale à l'or-
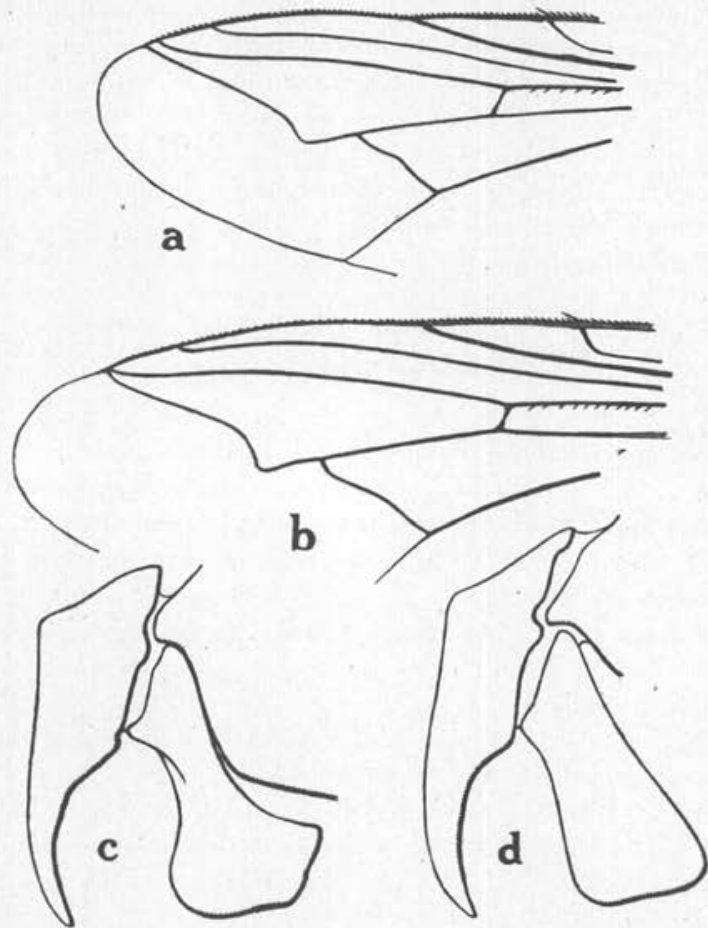

FIg. x. - Wohlfahrtia volucris et $W$. nuba. - $a$, extrémité de l'aile du $W$. volucris; $b$, id. du W. nuba; c, profil des branches des forceps externe et interne du $W$. nuba; $d$, id. du W. volucris.

bite; orbites et gênes d'un blanc d'argent. Soies frontales faibles. Gênes à ciliation microscopique éparse. Une soie verticale postérieure. Palpes roux à la base, brunis à l'apex. Antennes brunes, troisième article plus de deux fois plus long que le deuxième.

Thorax d'un gris blanchâtre. Mésonotum marqué de bandes longitudinales noirâtres. Chétotaxie : 3 humérales inégales; I sublatérale post-

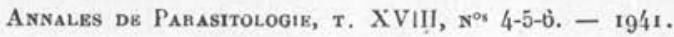


humérale; I présuturale; 2 notopleurales; I intra-alaire; 2 supra-alaires ; 4 dorso-centrales $(2+2)$; une paire d'acrosticales préscutellaires; scutellaires normales.

Patles à ciliation serrée, longue. Fémur II avec un peigne apical postérointerne formé de 10-12 épines courtes et robustes.

Aile : première cellule postérieure très étroitement ouverte à la marge ou fermée; quatrième section costale deux fois plus longue que la troisième; quatrième nervure : section discale (comprise entre les deux transverses) trois fois plus longue que la section préapicale entre la transverse et le coude (fig. $1, a$ ).

Abdomen : tergite II avec une tache médiane occupant toute la hauteur du segment et deux taches latérales rondes; tergite II avec une tache médiane triangulaire à pointe antérieure étendue sur toute la hauteur du tergite, deux taches latérales rondes placées au bord apical du segment; tergites IV et V avec trois taches rondes postérieures. Appareil copulateur roux; branches du forceps externe formées d'une plaque en massue étranglée à la base (fig. 1, c). - Longueur : 8,5-1 I mm.

Tunisie: Bou-Hedma, mai (C. Dumont).

Wohlfahrtia volucris n. sp.

бَ. Tête. - Espace interoculaire subégal au tiers de la largeur de la tête vue de face $(7: 2,5)$. Bande médiane frontale grise légèrement moins large que l'orbite à la base des antennes. Palpes entièrement roux. Antennes en grande partie d'un roux brun ; troisième article plus de deux fois plus long que le deuxième.

Chétotaxie thoracique : comme sur le $W$. nuba, sauf 3 supra-alaires, la seconde beaucoup plus forte; dorso-centrales $1+2$ ou $2+2$; acrosticales : deux paires préscutellaires.

Pattes à ciliation courte, peu serrée. Fémur II sans peigne spinuleux apical, les épines remplacées par de petites soies.

Aile : première cellule postérieure étroitement ouverte à la marge; quatrième section costale n'égalant pạs une fois et demie la troisième ; quatrième nervure : section discale n'égalant pas deux fois la section préapicale entre la transverse et le coude (fig. $\mathrm{i}, b$ ).

Abdomen avec les mêmes marques sombres que chez le $W$. nuba typique. Appareil copulateur roux; branches du forceps externe formées par une plaque rectangulaire non étranglée à la base (fig. $\mathrm{r}, d$ ). Longueur : $9 \mathrm{~mm}$.

Tunisie : Maknassy, septembre, capturé à la lumière d'une lampe (C. Dumont).

Le $W$. volucris se distinguera immédiatement du $W$. nuba par les fémurs II inermes; par sa chétotaxie hésitante; par la constitution de la tête (écartement des yeux, etc.). Le $W$. volucris est probable- 
ment une espèce jointive du W. naba. Un abondant matériel permettant des dissections en séries serait nécessaire pour définir les caractères des formes de passage.

On sait que les espèces jointives apparaissent spontanément chez certains Calliphorides à tendances parasitaires. L' " espèce " se disloque sous l'influence des réactions du parasitisme, ou à la suite d'un isolement étroit imposant des conditions de vie nouvelles. On sait que les Wohlfahrtia recherchent activement des actions parasitaires.

\section{Tableau des espèces.}

I-(2). Abdomen en grande partie ou complètement noir. Taches de pruinosité réduites ou nulles.

- Abdomen entièrement noir brillant . . . . W. atra Aldrich $0^{\Upsilon 7}$ ㅇ․

- Abdomen : tergites à très larges taches noires, confluentes. Taches de pruinosité très petites, mais bien définies . . . W. vigil Walker $0^{1}$ 우. 2-(I). Abdomen largement couvert de pruinosité, à lignes et taches noires bien définies par les tergites.

3 -(4). Espèce de grande taille. Pattes $\left(\sigma^{\top}\right)$ à villosité bien marquée. Tergites I-IV à taches noires nettes. Tèrgites $V$ entièrement couvert de pruinosité

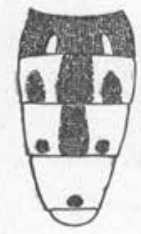

a

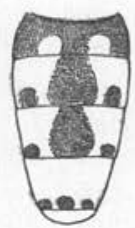

b

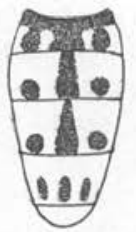

c

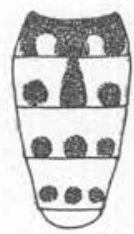

d

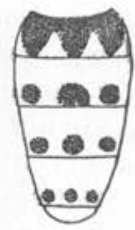

e

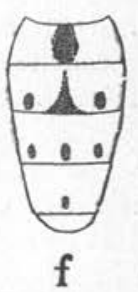

f

Fic. 2. - Ornementation abdominale des Wohljahrtia. - a, W. bella Macquart; $b, W$. magnifica Schiner; $c, W$. meigeini Schiner; $d, W$. trina W.; $e, W$. triquetra Séguy; $f, W$. seguyi Salem.

grise, sans taches manifestes (fig. 2, a). . . W. bella Macquart $\sigma^{\top}$. 4-(3). Espèce de taille moyenne. Tergites I-V tachés de noir.

5-(го). Tergite IV : tache centrale bien développée, étendue sur la ligne médiane jusqu’à l'apex du tergite III (fig. $2, c)$.

6-(7). Antennes : deuxième article roux orange, subégal au troisième article. Palpes roux . . . . . . . . W. meigeni Schiner $\sigma^{x}$.. 7-(6). Deuxième article de couleur brune; troisième article plus long que le second. Palpes noirs.

8-(9). Tergite III à taches latérales élargies, pointues et prolongées antérieurement jusqu'au bord postérieur du tergite II . W. triquetra Séguy $\sigma^{\nearrow}$. 
9-(8). Tergite III à taches latérales isolées, non prolongées jusqu'au bord antérieur (fig. 2, b).

- Dorso-centrales antérieures présentes . . W. magnifica Schiner $\sigma^{\top}$.

— Dorso-centrales nulles. . . . . . W. villeneuvei Salem $\sigma^{\top} 0$.

10-(5). Tergite IV : tache médiane de grandeur moyenne, triangulaire, ou ronde et isolée au bord postérieur (fig. $2, d$ ).

I I-(12). Antennes : troisième article subégal au deuxième, d'un roux orange. Tibias II et III avec une villosité bien développée. Tergite IV : tache centrale isolée, mais avec un prolongement linéaire antérieur.

I2-(1 1). Troisième article plus long que le deuxième.

13-(14). Troisième article n'atteignant pas deux fois la longueur du second. Bandes mésonotales élargies, noires. Tergites à marques latérales rondes, élargies; tergite II noirci par confluence des taches (r).

W. pachytyli Tnsd. $O^{x}+$.

14-(13). Troisième article antennaire au moins deux fois aussi long que le deuxième.

15-(28). Mâles.

I6-(17). Fémur II sans peigne apical postéro-interne. Abdomen : taches tergales de grandeur normale, chatoyantes.

- Aile : quatrième nervure aboutissant à une certaine distance de l'apex de l'aile.

W. pattoni Salem $\sigma^{x}$.

- Quatrième nervure aboutissant normalement dans la région préapicale de l'aile . . . . . . . . . . . . . . . volucris Séguy.

17-(16). Fémur II avec un peigne apical postéro-interne formé d'épines courtes. Taches tergales nettes, non ou peu chatoyantes.

18-( I 9$)$. Aile : troisième et quatrième sections costales subégales. Tibias II et III villeux. Phallus avec un processus épineux externe.

W. trina Wied. $\sigma^{x}$.

I9-(r8). Aile : quatrième section costale une fois et demie aussi longue que la troisième. Phallus sans processus dorsal.

20-(2I). Palpes bruns ou roux, leur extrémité brune ou grise. Antennes : deuxième article d'un gris sombre sauf une légère teinte orange sur la partie apicale et médiane. Sternites et pattes couverts d'une longue villosité.

- Abdomen : taches tergales latérales grandes. Antennes : troisième article trois fois plus long que le deuxième. . . . . . W. indigens Vill.

- Taches tergales petites (fig. $2, f$ ). Antennes : troisième article quatre fois plus long que le deuxième. . . . . . . . W. seguyi Salem (2) 21-(20). Palpes d'un roux orange.

22-(23). Antennes : deuxième article brun. Pattes nues.

(1) W. pachylyli Townsend 1918 , Ins. Ins. Mens., VI, p. $159=$ Disjuncto brunnipalpis Bezzi, 1908 (nec Macquart) $=W$. euvittala Villeneuve, 1920. - Transvaal ex Pachytylus sulcicollis ex C. W. Howard ap. Townsend.

(2) Diffère du $W$. villeneuvei par la conformation de l'appareil copulateur. 
23-(22). Deuxième article orange. Pattes à villosité serrée.

24-(25). Forceps internes à branches très épaisses à la base, courtes, triangulaires

W. erythrocera Villen. 25-(24). Forceps internes médiocrement épaissis.

26-(27). Phallus sans apophyse externe . . W. indigens æthiopica Villen. 27-(26). Phallus avec une apophyse externe plus ou moins développée.

- Tergite III avec une tache médiane triangulaire occupant toute la hauteur du segment

W. nuba W.

- Tergite III avec une tache médiane isolée à l'apex du segment.

„8-( 15$)$. Femelles.

29-(30). Abdomen : taches tergales très réduites; tergite II avec seulement une tache centrale noire.

W. brunnipalpis Macq. 3o-(29). Taches tergales non réduites, bien délimitées sur tous les segments. 3r-(32). Tergite III : tache centrale isolée, ronde, sans prolongation antérieure (fig. $2, e$ ).

- Petite nervure transverse très rapprochée du niveau de l'apex de la souscostale. Antennes rousses.

W. seguvi Salem.

- Petite transverse placée largement après le niveau de l'apex de la souscostale. Antennes noires

W. triquetra Séguy.

$3_{2}-\left(3_{1}\right)$. Tergite III : tache centrale plus ou moins étalée antérieurement jusqu'au bord postérieur du tergite II.

33-(34). Aile : deuxième section costale subégale à la troisième ou très légèrement plus longue. Antennes et palpes bruns . . . . W. trina W.

34-(33). Aile : deuxième section costale au moins une fois et demie aussi longue que la troisième. Antennes : deuxième article et palpes d'un roux orange.

35-(36). Aile : section proximale de la nervure 4 située entre les deux transverses au moins trois fois aussi longue que la section préapicale située près du coude. Abdomen d'un gris bleuâtre à taches chatoyantes noires.

36-(35). Section proximale de la nervure 4 moins de trois fois aussi longue que la section préapicale. Abdomen en majeure partie gris ou gris verdâtre.

$37-(40)$. Antennes : troisième article deux fois et demie (ou plus) aussi long que le deuxième.

38-(39). Espace interoculaire tout au plus aussi large que l'œil vu de face. Palpes jaunes.

W. erythrocera Villen.

$39-(38)$. Espace interoculaire plus large que l'œil.

- Antennes et palpes brunis

W. indigens Vill.

40- $(37)$. Troisième article tout au plus deux fois aussi long que le deuxième.

- Quatrième nervure : section comprise entre les deux transverses n égalant pas trois fois la longueur du segment préapical situé avant le coude.

- plus de trois fois.

W. smarti Salem.

W. nuba Wied. 


\section{Bibliographie}

Bunnchard (R.). - Un cas de myiase par la Sarcophaga magnifica en Roumanie. Bull. Soc. Zool. France, XVI, r891, p. 25-26.

Brundr (E.). - Larven der Wohlfarts'chen Fliege (Sarcophila wohlfartii Portseh.) im Zahnfleische des Menschen. Zool. Anz., XI, 1888, p. 56o.

Brumpt (E.). - Précis de parasitologie. $5^{e}$ édition, Paris (Masson), r936, XII-21 39 p., , 1085 fig., $4 \mathrm{pl}$.

Condonelli (F. M.). - Miiasi auricolare par Sarcophaga carnaria. Bull. Accad. Gioenia, Catania, fasc. XXVIII, 1913, p. 7-10.

- Ancora sulla miliasi auricolare (Sarcophaga magnifica, Lucilia macellaria, Calliphora vomitoria, Anthomyia pluvialis). Bull. Accad. Gioenia, Catania, fasc. XXXI, 19r4, p. 15-23.

Cros (A.). - Parasitisme chez l'Homme des larves de Wohlfahrtia (Sarcophila) magnifica Schiner. Ball. Soc. Hist. Nat. Ajrique du Nord, I, r910, p. 54-55.

Deranoë (P.). - Myiases du bétail du Cercle des Doukkala causées par les larves d'une mouche sarcophile, Wohlfartia magnifica Schiner 1862 . Bull. Soc. Sc. nat. du Maroc, II, 1922, p. 132-136, fig. I

Dubors (M.). - Un Diptère parasite de l'homme (Sarcophila wohlfartii Portsch.). Bull. Soc. Linn. Nord de la France, III, $1876-1877$, p. $241-245$.

Fammame (L.). - Sur des larves d'Anthomyia extraites de l'oreille d'un malade. Ann. Soc. Ent. France (5), VI, 1876, Bull. p. xxir.

Faure (J. C.). - The Life History of the Red Locust. Bull. Dep. Agric. S. Afr., Pretoria, $\mathrm{n}^{\circ} \mathrm{r} 44, \mathrm{r} 935,32 \mathrm{p} ., 5 \mathrm{pl}$. I carte.

Frrarène (C.). - Descriptions de deux importants Chalcidiens d'Égypte et du Soudan. Bull. Soc. R. ent. Egypte, XIX, 1935, p. 365-37o, 3 fig.

Fond (N.). - Observations on the Behaviour of the Sarcophagid Fly, Wohlfahrtia vigil (Walk.). Jl. Parasit., XIX, 1933, p. ro6-111.

Further Observations on the Behaviour of Wohljahrtia vigil (Walk.) with Notes on the Collecting and Rearing of the flies. $J l$. Parasit., XXII, r936, p. $309-328$, 4 fig., $x$ carte.

Francaviglia (M. C.). - Ancora sulla mjiasi auricolara. Boll. Sedute Accad. Gioenia, Catania, $\mathrm{n}^{\circ} 3 \mathrm{r}, \mathrm{19} \mathrm{r} 4$, p. $15-23$.

Gerstäcker (A.). - Ueber das Vorkommen ausgewachsener lebender Dipterenlarven in den Nasenhöhle des Menschen. Sitzber. d. ges. Naturf. Freunde, Berlin, 1875, p. 108-110.

Gertson (C. D.), Lancaster (W. E. G.), Larson (G. A.) et Wheeler (G. C.). Wohlfahrtia myiasis in North Dakota. Report of two Cases. Jl. Amer. Med. Ass., $1933, \mathrm{n}^{\circ} 7$, p. $487-488$.

Goldschmid (W.). - Einige Bemerkungen zur Frage der Ophthalmomyiasis. Wiener Klin. Wochenschr., XXXII, 1919, no 48, p. Ir59-1 60 . Analyse dans Trop. Dis. Bull., XVI, 1920, p. rog.

Govgr (L.). - On Wohrfahrtia magnifica, a Sarcophagid parasiting Man. Bull. Soc. ent. Egypte, 1917 , p. 23-25.

Grantham-Hits (C.). - Preliminary Note on the Treatment of infected Wounds with the Larva of Wohljahrtia nuba. Trans. R. Soc. Trop. Med. Hyg., XXVII, r933, p. $9^{3 \cdot 98}$, r pl.

HAGEN (H. A.). - Note on a Sarcophagous larva found in a girl's neck. Proc. Boston Soc. Nat. Hist., XX (1878-1880), 1881, p. 409-410.

Jausion (H.) et Dekester (M.). - Sur la transmission comparée des kystes d'Enta- 
mœba dysenteriæ et de Giardia intestinalis par les mouches. Arch. Inst. Pasteur Afr. N., III, r923, p. 154-155.

JoHanssen (O. A.). - The first Instar of Wohlfahrtia vigil Walker. Jl. Parasito$\log y$, VII, 1921 , p. 154-155.

Kingscote (A. A.). - Myiasis in Ranch-raised Foxes. 62nd Ann. Rep. Ent. Soc. Ontario, 1931, p. 91-93.

Laboulbène (A.). - Observations de myiasis dues à la Sarcophaga magnifica Schiner. Ann. Soc. ent. France, (6), IV, I884, p. 28 , pl. I, fig. 7 .

Lewis (L.). - Larvæ (of fly) in the external ear. Brit. Med. Journ., I873, II, p. 370 .

Lurse. - Durch Fliegen und ihre Larven verursachte Erkrankungen. Deutsch. Tierärzt. Wochenschr., XXIII, x915, $\mathrm{n}^{\circ} 46$, p. $395-397,7$ fig.

Macias y Macias (F.). - Otro caso de otomiasis por Wohlfahrtia magnifica (Schiner, 1862). Med. Paises calidos, VIII, r935, p. 538-541.

Maxkiewicz. - Ueber das Vorkommen von Fliegenlarven in den Nasenhöhle. Arch. $f$. path. Anat. $u$. Phys., XLIV, 1868 (4 f, 4 Bd.), p. 375 .

MÉGNiv (P.). - Sur les diptères qui pondent dans les plaies des animaux vivants. Ann. Soc. Ent. France (5), VIII, 1878 , Bull., p. III-v.

Morrison (F. O.). - Myiasis in the Foot of a Rat by Wohlfahrtia meigeni Schiner. Canad. Ent., LXIX, ${ }_{93} 37, \mathrm{n}^{\circ}$ 12, pp. 266-269.

Najera Angulo (L.). - Primer caso de otomiasis por Wohlfahrtia magnifica descrito en España. Med. Paises calidos, VIII, r935, p. 469-474, 4 fig.

Onorato (R.). - Le miasi in Tripolitania. Arch. Ital. Sc. Med. Colon., III, 1922, $\mathrm{n}^{\mathrm{os}}$ I-12.

Patron (W. S.). - Notes on the myiasis-producing Diptera of man and animals. Bull. Ent. Res., XII, 1922, p. 239-26I (192I-1922).

Pavlovskis (E. N.). - Ueber miasen in Turkmenien. Trud. Sov. Izuch. proizv. Sil., Ser. Turkmensk., pt. 6, 1934, p. 119-140, 6 fig.

Perroncito (E.). - Le larve della Sarcophila magnifica Schiner nell'intestino dell'uomo. Giorn. Acc. Med. Torino, LXIII, 190o, p. 522-525, pl. Monit. Zool. Ital., XI, p. $28 \mathrm{r}$.

Popov (L. V.). - A Case of Myiasis in Man caused by the Larvæ of Wohlfahrtia magnifica. Trop. Med. Vet., IX, 1931, $\mathrm{n}^{\circ} 6$, p. $288-289$ (en russe, sommaire en anglais).

Portchissky (J.). - Materialien zur Naturgeschichte der Fliegen und ihrer Larven, welche Krankheiten bei Menschen und Thieren veranlassen, mit einem Uberblick über die Erscheinungen der Myiasis. Horæ Soc. Entom. Ross., IX, $1875-1876$, p. 3-1 80 ( 1875$)$.

- Krankheiten welche im Mohilew'schen Gouvernement von den Larven der Sarcophila wohlfahrti entstehen und deren Biologie. Horæ Soc. ent. Ross., XI, 1875, p. 123-162, pl. III, IV, V.

- Sarcophilæ wohlfahrti monographia. Horæ Ent. Ross., XVIII, 1884, p. 247 . 3 r4.

- Wohljahrtia magnifica Schin., and allied Russian species. The biology of this fly and its importance to man and domestic animals. Memoirs Bureau of Entomology, Scientific Committee, Ministry of Agriculture, Petrograd, XI, 1916, ro 8 p., 39 fig., 2 pl. Analyse dans Rev. Appl. Entom., Sér. B, IV, 1916 , p. 88.

Ruch (C. L.) et Knowrton (G. F.). - Myiasis of Baby in Utah. Jl. Econ. Ent., $\mathrm{XXX}$, I937, p. 678 .

Roubaud (E.). - Etudes sur la faune parasitaire de l'Afrique occidentale française. 
I. Les producteurs de myiases et agents similaires chez l'homme et les animaux. Paris, Larose, 1914, p. 1-251, 70 fig., 4 pl.

SAlem (H. H.). - A complete revision of the species of the genus Wohlfahrtia B. et B. Egyptian Univ. Fac. of Medecine, Publ. n ${ }^{\circ}$ 13, 1938, p. I-9o, 26 fig., 3 pl.

Selezneva (M. V.). - A Case of Infestation of the auditory Duct by Wohljahrtia in the environs of Perm (en russe). Med. Parasit., V, $1936, \mathrm{n}^{\circ} 4$, p. 632.

Shannon (R. C.). - Non-human Host Records of Wohlfahrtia. Proc. Ent. Soc. Wash., XXV, 1923, p. 142 .

Taschenberg (E.). - Larven einer Sarcophaga aus den Menschlichen Ohr. Ztschr. f. d. Ges. Naturw., XXXVI, 1870 , p. 447.

Townsend (C. H. T.). - New Muscoid genera, species and synonymy. Ins. Insc. Mens., VI, 1918, $\mathrm{n}^{\mathrm{os}}$ 10-12, p. $157-182$.

Volkovicz (E. N.). - Les larves de Wohlfahrtia magnifica Schin. aux dents et aux gencives de l'homme en Russie et les modes populaires de les en extraire. Trd. b. entom. ucen. kom. gl. Upr. Zeml., St. Petersburg, IX, rgir, p. $x-15$.

Walker (E. M.). - Wohlfahrtia vigil (Walker) as a Human Parasite. Jl. Parasito$\log y$, VII, 1920, p. I-I 7 .

- Some cases of cutaneous Myiasis, with notes on the larvæ of Wohlfahrtia vigil (Walker). Jl, Parasit., IX, I922, p. 1-5, 3 pl.

- Cutaneous Myasis in Canada. Canad. Publ. Hlth. Jl., I93r, p. 5o4-5o8, 2 fig.

- The Larval Stages of Wohljahrtia vigil (Walker). Jl. Parasit., XXIII, 1937 , p. $163-174,24$ fig.

Laboratoire d'Entomologie du Muséum d'Histoire naturelle de Paris.

(Directeur : Professeur R. Ieannel). 